\title{
Conic quantization: stochastic volatility and market implied liquidity
}

\author{
Lucio Fiorin* Wim Schoutens ${ }^{\dagger}$
}

August 27, 2019

\begin{abstract}
In this paper we introduce a new technology for the pricing of European options for a wide class of models. The method is based on a quantization technique that exploits the knowledge of the characteristic function for the price process in closed form, and is quick and accurate enough to calibrate on financial data. Moreover, it allows to introduce the concept of market implied liquidity, linking the pricing under stochastic volatility with the Conic Finance theory of two prices. As a motivating example, we construct for the first time the market implied liquidity surface under stochastic volatility.
\end{abstract}

Keywords: Quantization, characteristic function, conic finance, option pricing, stochastic volatility, implied liquidity.

\section{Introduction}

The paradigm of frictionless markets is the base for classical option prices theories, especially when considering numerical methods for the pricing of derivatives. Financial market, however, present drops in liquidity, especially during stress periods. In this paper we will consider liquidity in terms of the spread between the bid price and the ask price of an option.

The literature that studies and measures bid-ask spreads in financial markets is quite variegate. This feature has been tackled mostly in two different ways. The first one follows an approach that considers the introduction of transaction costs, see for example Davis et al. (1993), Shreve and Soner (1994), Cvitanic and Karatzas (1996), Barles and Soner (1998). This approach looks promising, but not flexible enough to capture the magnitude of the spread observed in the markets, see Leippold and Scharer (2017). A second one, introduced in Cherny and Madan (2009) and developed in Madan and Cherny (2010), is based on the theory of conic finance. The idea behind this approach, that we will use as a starting point in this paper, is that the market sells and buy derivatives from and to financial investors, and investors buy at ask price and sell at bid price. The market is then seen as a central counterparty that carries out all the acceptable trades, based on acceptability indices, which comes from the theory of coherent risk measures of Artzner et al. (1999).

The theory of Conic Finance proposes to model market illiquidity using a market stress level parameter, and the bid-ask spread is a consequence of the acceptability indices assigned by the market, in terms of distortion of the risk-neutral pricing measure. This approach assumes a static liquidity model, and has been extended also in Corcuera et al. (2012) and Albrecher et al. (2013). In these works, the authors assumed that the underlying stock price has a Black Scholes (in Corcuera et al. (2012)), or Levy with closed form density (in Albrecher et al. (2013)), dynamics. The liquidity parameter given by the market, defined as implied liquidity, has a stochastic behaviour, and has been generalized in Leippold and Scharer (2017). We contribute to the literature of liquidity modelling for the pricing of option in different ways. First, we introduce the concept of uniform quantization for the pricing of options under stochastic volatility. Quantization is a tool coming from signal processing, and consists in finding a discrete random

\footnotetext{
*Department of Mathematics "Tullio Levi Civita", University of Padova, via Trieste 63, 35121 Padova, Italy. Email: luciofiorin@gmail.com

${ }^{\dagger}$ Department of Mathematics, Celestijnenlaan 200 B, B-3001 Leuven Belgium. Email: wim@schoutens.be
} 
variable approximating in the best possible way a continuous random variable. Using some ideas from Fourier analysis, it is possible to compute the (optimal) quantization of a stochastic process in a fast and accurate way. This quantization technique can be used, together with Conic Finance, to compute bid and ask prices for European put and call options in every model having a characteristic function in closed form. To the best of our knowledge, this is the first paper that studies the pricing of options considering the bid-ask spread under stochastic volatility for the underlying. In addition, we use this pricing tool to compute the surface of implied liquidity generated in presence of stochastic volatility. In the previous works of Corcuera et al. (2012), Albrecher et al. (2013) and Leippold and Scharer (2017), implied liquidity was considered only for Black Scholes type of dynamics.

The paper is organized as follows: in Section 2 we recall some basic notions on quantization, introducing the main results and the motivation behind this technique. In Section 3 we recall some notions on Conic Finance, especially in the distortion of the distribution density of the price. In Section 4 we introduce the new quantization algorithm, and we connect Quantization and Conic Finance. In Section 5 we show the performance of the method on pricing options and bid-ask spreads. Finally, in Section 6 we present a calibration exercise on market data, and we introduce the concept of implied liquidity under stochastic volatility. Section 7 concludes.

\section{Brief overview on quantization}

Quantization is a tool widely used in numerical probability, and it can be seen as a compression technique for the approximation of stochastic processes and random variables. The idea behind the quantization of a random variable $X$ in $\mathbb{R}^{d}$ is to find the best approximation using only a finite number of points, therefore creating a discrete random variable as close as possible to $X$. If, for example, one wants to find the discrete random variable that takes only one value, which minimize the distance from $X$, where we consider as distance the $L^{2}$-norm, straightforward computations show that the $\mathbb{E}[X]$ is exactly the value that one has to take. But if we consider a discrete random variable $\widehat{X}$ that takes $N$ values, where $N>1$, we have to take into consideration also the construction of the distribution of $\widehat{X}$.

Before going into details about how to construct this discrete random variable $\widehat{X}$, that we will call quantizer of $X$, let us see the consequences of such an approximation. We assume that $\widehat{X}$ takes values in a set $\Gamma_{N}$ of cardinality $N, \Gamma_{N}=\left\{x_{1}, \ldots, x_{N}\right\}$. In fact, in view of the pricing of a derivative product, integrals of the form $\mathbb{E}[h(X)]$, where $h: \mathbb{R}^{d} \rightarrow \mathbb{R}$ is a Borel function, can be approximated as

$$
\mathbb{E}[h(X)] \cong \mathbb{E}[h(\widehat{X})]=\sum_{i=1}^{N} h\left(x_{i}\right) \mathbb{P}\left(\widehat{X}=x_{i}\right) .
$$

Quantizing $X$ on a given set of points $\Gamma_{N}=\left\{x_{1}, \cdots, x_{N}\right\}$, also called quantization grid, boils down to projecting $X$ on the grid $\Gamma_{N}$ following the closest neighbour rule. An $N$-quantizer is a Borel function $f_{\Gamma_{N}}: \mathbb{R}^{d} \rightarrow \Gamma_{N} \subset \mathbb{R}^{d}$ projecting $X$ on $\Gamma_{N}$. We can then compute the $L^{2}$ distance between $X$ and $\widehat{X}$, that reads

$$
\left\|X-f_{\Gamma_{N}}(X)\right\|_{2}=\left\|\min _{1 \leq i \leq N}\left|X-x_{i}\right|\right\|_{2}
$$

where $\|X\|_{2}:=\left[\mathbb{E}\left(|X|^{2}\right)\right]^{1 / 2}$ is the usual norm in $L^{2}$. As a function of the grid $\Gamma_{N}$, the $L^{2}$ distance, also called quantization error is continuous and reaches a minimum over all the grids with size at most $N$, see Graf and Luschgy (2000), Pagès (2015). It is then possible to find the set of points $\Gamma_{N}^{\star}$ of size $N$ which minimizes the quantization error, in order to find the best approximation of $X$ in the $L^{2}$ sense. We will then call $\widehat{X}=f_{\Gamma_{N}^{\star}}(X)$ the optimal quantizer of $X$. Please note that $\Gamma_{N}^{\star}$ may not be unique, so $\widehat{X}$ is an optimal quantizer of $X$. Every optimal quantizer $\widehat{X}$ is associated to an optimal grid of points $\Gamma_{N}^{\star}$, and viceversa, and also to an optimal Borel partition of the space $\mathbb{R}^{d},\left(C_{i}\left(\Gamma_{N}^{\star}\right)\right)_{1 \leq i \leq N}$, and viceversa, so that the quantizer is defined as follows

$$
f_{\Gamma_{N}^{\star}}(X)=\sum_{i=1}^{N} x_{i} \mathbb{1}_{C_{i}\left(\Gamma_{N}^{\star}\right)}(X),
$$


where the above partition $\left\{C_{i}\left(\Gamma_{N}^{\star}\right)\right\}_{i=1, \ldots, N}$, with $C_{i}\left(\Gamma_{N}^{\star}\right) \subset\left\{\xi \in \mathbb{R}^{d}:\left\|\xi-x_{i}\right\|=\min _{1 \leq j \leq N}\left\|\xi-x_{j}\right\|\right\}$, is called the Voronoi partition, or Voronoi tessellation induced by $\Gamma_{N}^{\star}$.

In addition, the quantization error vanishes as the grid size goes to infinity and the convergence rate has been obtained via the Zador theorem (see Graf and Luschgy (2000)):

$$
\min _{\Gamma_{N},\left|\Gamma_{N}\right|=N}\left\|X-f_{\Gamma_{N}}(X)\right\|_{2}=Q\left(\mathbb{P}_{X}\right) N^{-1 / d}+O\left(N^{-1 / d}\right)
$$

where $Q\left(\mathbb{P}_{X}\right)$ is a positive constant. The interesting result of this Theorem is the rate of convergence of the optimal quantizer. In fact, when we are considering the approximation of one dimensional random variables, as it will be the case in our option pricing exercise, the error convergence is linear, in comparison with the Monte Carlo error convergence which is of order $o\left(\frac{1}{\sqrt{N}}\right)$, where $N$ is the number of simulations. Quantization in finance has been studied broadly, especially in the works of Pagès and Sagna (2015), Callegaro et al. (2015) and McWalter et al. (2018) for local volatility models, and Callegaro et al. (2016), Fiorin et al. (2018) and Callegaro et al. (2018) for stochastic volatility models.

\section{Conic Finance and the bid - ask spread pricing}

In this section we recall some basic notions on the theory of Conic Finance, when considering the pricing of the bid-ask spread of an option. Throughout the paper we will make the assumptions of zero cost cash flows and constant interest rate factor $r$.

Let us consider the case of the pricing of a European Call option on the underlying $S$, with strike $K$ and maturity $T$. The case of a Put option, or of more exotic options can be easily generalized. One person can be interested in being long a Call, or alternatively being short a call. In the case of a long position, one would typically look at zero cost cash flows of the following form:

$$
\left(S_{T}-K\right)^{+}-e^{r T} b
$$

in the case where one agrees to pay at time $T$ a cash amount $e^{r T} b$, i.e., equivalently, a cash amount $b$ at time 0 , and agrees to receive the payoff. In alternative, in case of a short position, the cash flow would be of the form:

$$
e^{r T} a-\left(S_{T}-K\right)^{+},
$$

in the case where one agrees to receive at time $T$ a cash amount $e^{r T} a$, i.e., equivalently, a cash amount $a$ at time 0 , and agrees to payout the payoff. We will call $b$ the bid price, that is the price the market wants to pay for the risk $\left(S_{T}-K\right)^{+}$, and $a$ the ask price, the price that the market wants to receive for the risk $\left(S_{T}-K\right)^{+}$. Any price higher than $b$ and any price lower than $a$ will make these prices unacceptable. Under this construction, the risk neutral price will be lower than the ask price and greater than the bid price.

In fact, let us consider the set of zero cost cash flows $Z$ defined as

$$
\mathcal{A}^{*}=\left\{Z \mid e^{-r T} \mathbb{E}^{\mathbb{Q}}[Z] \geq 0\right\},
$$

where $\mathbb{Q}$ is the risk neutral distribution. The set $\mathcal{A}^{*}$ is a half space containing all the non-negative random variables. This means that this set contains all the possible arbitrages, but one needs to take into consideration also zero cost cash flows that are not necessary arbitrages, in this set of acceptable cash flows. Note that the set of non-negative random variables constitutes a convex cone, and also $\mathcal{A}^{*}$ is a convex cone. The half space $\mathcal{A}^{*}$ is then too big for our purposes, and in the two price economy one would consider a set $\mathcal{A}$ for the acceptable zero cost cash flows such that $\mathcal{A}$ contains all the arbitrages and $\mathcal{A} \subset \mathcal{A}^{*}$.

It is shown in Artzner et al. (1999) that the elements of $\mathcal{A}$ can be defined by a convex set of probability measures $\mathcal{M}$ where

$$
Z \in \mathcal{A} \Longleftrightarrow e^{-r T} \mathbb{E}^{Q}[Z] \geq 0 \quad \text { for all } Q \in \mathcal{M} .
$$


The market accepts the risk to buy at the best bid price $b$ and the risk to sell at the best ask price $a$. This means that the zero cost cash flows $\left(S_{T}-K\right)^{+}-e^{r T} b$ and $e^{r T} a-\left(S_{T}-K\right)^{+}$are acceptable. Equivalently this means that for all $Q \in \mathcal{M}$

$$
\left(S_{T}-K\right)^{+}-e^{r T} b \in \mathcal{A} \Longleftrightarrow b \leq e^{-r T} \mathbb{E}^{Q}\left[\left(S_{T}-K\right)^{+}\right],
$$

and

$$
e^{r T} a-\left(S_{T}-K\right)^{+} \in \mathcal{A} \Longleftrightarrow a \geq e^{-r T} \mathbb{E}^{Q}\left[\left(S_{T}-K\right)^{+}\right] .
$$

This construction proves the intuition that the risk neutral price of a European Call option will be between the bid and the ask price.

Finally, the best bid and the best ask price at time zero for the European call are given by

$$
\operatorname{bid}\left(\left(S_{T}-K\right)^{+}\right)=e^{-r T} \inf _{Q \in \mathcal{M}} \mathbb{E}^{Q}\left[\left(S_{T}-K\right)^{+}\right],
$$

and

$$
\operatorname{ask}\left(\left(S_{T}-K\right)^{+}\right)=e^{-r T} \sup _{Q \in \mathcal{M}} \mathbb{E}^{Q}\left[\left(S_{T}-K\right)^{+}\right] .
$$

We refer to this construction as Conic Finance, since the prices are defined by convex cones of acceptable zero cost cash flows.

A market model can then be constructed giving a specification of a set of supporting measures, and the bid and ask prices can be computed as in (2) and (3). In Cherny and Madan (2009) the authors define operational cones, and make use of concave Conic distortion function in order to construct bid and ask prices for European options. A concave distortion function is a concave function which maps the unit interval to itself. In this work we will use only the MINMAXVAR Conic distortion function defined as

$$
\Psi_{\lambda}(u)=1-\left(1-u^{\frac{1}{\lambda+1}}\right)^{1+\lambda}
$$

where $\lambda$ is a positive real value.

The interested reader is referred to Madan and Schoutens (2016) and reference therein for further details.

\section{The uniform Fourier quantization algorithm}

In this section we present a new technique for the quantization of the underlying of an option at maturity, and we link it with the Conic pricing introduced in Section 3 . The link between Fourier analysis and quantization techniques has been already studied and implemented in Callegaro et al. (2018). In this paper we use a different approach, in order to improve the efficiency of the pricing algorithm, as the numerical results in Section 5 will show.

Let us consider a probability space $(\Omega, \mathcal{A}, \mathbb{P})$ and an $\mathbb{R}$-valued random variable $X$. Let us call $F$ the distribution function of $X$, i.e. $F(x)=\mathbb{P}(X \leq x), \quad x \in \mathbb{R} . F$ is always non-decreasing, càdlàg and such that $\lim _{x \rightarrow-\infty} F(x)=0$ and $\lim _{x \rightarrow+\infty} F(x)=1$, and it is possible to associate to $F$ the canonical left inverse $F_{l}^{-1}$ :

$$
F_{l}^{-1}(u)=\inf \{x \mid F(x) \geq u\} .
$$

Then $F_{l}^{-1}(u)$ is non decreasing, left continuous and satisfies $F_{l}^{-1}(u) \leq x \Longleftrightarrow u \leq F(x)$. It is then immediate to see that, if $U$ is a uniform random variable in $[0,1]$, then $F_{l}^{-1}(U) \stackrel{d}{=} X$. This means that, for every measurable function $h \in L^{1}\left(\mathbb{P}_{X}\right)$, the following holds:

$$
\mathbb{E}[h(X)]=\mathbb{E}\left[h\left(F_{l}^{-1}(U)\right)\right] .
$$

This result is known as the Fundamental Theorem of simulation, and it will be essential for the quantization algorithm proposed in this paper. 


\subsection{Optimal quantization of uniform random variables}

The link between the distribution of a generic random variable and (a modification of) the distribution of a uniform random variable has important applications also in quantization. It is easy to prove, see Graf and Luschgy (2000, Example 4.17), that the optimal quantization grid of size $N, \Gamma_{N}^{*}=\left(u_{1}, \ldots, u_{N}\right)$, for a uniform random variable $U \sim U([0,1])$ is given by

$$
u_{j}=\frac{2 j-1}{2 N}, \quad j=1, \ldots, N .
$$

Let us define the optimal quantizer $\widehat{U}^{\Gamma_{N}^{*}}$ of $U$ in the canonical way, i.e.,

$$
\widehat{U}^{\Gamma_{N}^{*}}=\sum_{j=1}^{N} u_{j} \mathbb{1}_{C_{j}\left(\Gamma_{N}^{*}\right)}(U),
$$

where $C_{j}\left(\Gamma_{N}^{*}\right)$ is the Voronoi cell associated to $u_{j}$, namely $C_{j}\left(\Gamma_{N}^{*}\right)=\left[\frac{j-1}{N}, \frac{j}{N}\right], \quad j=1, \ldots, N$. Note that, because of the geometric construction of the Voronoi cells, it follows immediately that

$$
\mathbb{P}\left(\widehat{U}^{\Gamma_{N}^{*}}=u_{j}\right)=\mathbb{P}\left(U \in C_{j}\left(\Gamma_{N}^{*}\right)\right)=\frac{1}{N} \quad \forall j=1, \ldots, N .
$$

Motivated by these results, we can approximate the expectation of a generic measurable function $g$ : $[0,1] \rightarrow \mathbb{R}$ in the following way:

$$
\mathbb{E}[g(U)] \approx \sum_{j=1}^{N} g\left(u_{j}\right) \mathbb{P}\left(U \in C_{j}\left(\Gamma_{N}^{*}\right)\right)=\frac{1}{N} \sum_{j=1}^{N} g\left(u_{j}\right),
$$

so the expectation in (4) can be approximated, setting $g=h \circ F_{l}^{-1}$, as

$$
\mathbb{E}[h(X)]=\mathbb{E}\left[h\left(F_{l}^{-1}(U)\right)\right] \approx \frac{1}{N} \sum_{j=1}^{N} h\left(F_{l}^{-1}\left(u_{j}\right)\right) .
$$

The study of the error boils down to the study of the convergence of the optimal quantizer to the original random variable. As seen in Section 2, due to the Zador Theorem, it is possible to have an a priori estimation of the convergence, which is linear. In Section 4.4 we give a more detailed estimation of the quantization error in the pricing of options. This is a reason why quantization should be preferred to Monte Carlo simulations, where the rate of convergence is of order $O\left(\frac{1}{\sqrt{N}}\right)$, where $N$ is the number of simulations.

\subsection{The uniform Fourier quantization approach}

Let us consider now the problem of the derivation of $F_{l}^{-1}$ in a closed form or, at least, in an efficient way. From now on we will consider $X$ as the value of the underlying $\log$ price process $S$ at maturity $T$, and $h$ as the payoff of a derivative at maturity (for example, in the case of a call with strike $K$, $\left.h(x)=\left(e^{x}-K\right)^{+}\right)$.

In the case where $S$ follows a Black Scholes dynamic, it is possible to compute the distribution function $F$ of the $\log$ process at time $T$ using the $\log$ normal property of $S$, but this is not always possible when we consider more complicated models, such as affine or Lévy processes. We will focus on processes for which the characteristic function can be easily computed.

Suppose that the characteristic function of the log process

$$
\phi_{T}(u):=\mathbb{E}\left[e^{i u \log \left(S_{t}\right)}\right], \quad u \in \mathbb{R}
$$

is known in closed form, or it can be easily computed. We omit, for the sake of clarity, the dependence from the initial value of the price process at time 0 . 
The characteristic function can be seen as the Fourier - Laplace transform of the density of the price process, so it can be proved (see e.g. Shephard (1991)) that

$$
F(x)=\mathbb{P}\left(\log \left(S_{T}\right) \leq x\right)=\frac{1}{2}-\frac{1}{\pi} \int_{0}^{+\infty} \operatorname{Re}\left(\frac{e^{-i u x} \phi_{T}(u)}{i u}\right) d u .
$$

If we assume enough regularity of the characteristic function $\phi_{T}(u)$, the distribution function of the $\log$ price $F(x)$ is continuous, so the canonical left inverse is exactly the inverse. Up to our knowledge it is not possible to write $F^{-1}$ in a closed form using the characteristic function $\phi_{T}$, but we can use a numerical technique to invert $F$.

As shown in Equation (5), the problem of the pricing of a European derivative can be approximated as

$$
\mathbb{E}[h(X)] \approx \frac{1}{N} \sum_{j=1}^{N} h\left(F^{-1}\left(u_{j}\right)\right),
$$

where $u_{j}=\frac{2 j-1}{2 N}$. We are then interested in the computation of

$$
y_{j}:=F^{-1}\left(\frac{2 j-1}{2 N}\right) \quad \forall j=1, \ldots, N .
$$

The determination of the values of the vector $\mathbf{y}=\left(y_{1}, \ldots, y_{N}\right)$ boils down to solve the following system:

$$
G_{j}(\mathbf{y}):=F\left(y_{j}\right)-\frac{2 j-1}{2 N}=0, \quad j=1, \ldots, N .
$$

This system can be easily solved computing the function $F$ in a fine grid of points, and then the solution $\mathbf{y}=\left(y_{1}, \ldots, y_{N}\right)$ can be obtained via interpolation. This approach is much faster than the Newton Raphson algorithm implemented in Callegaro et al. (2018). In fact we are able to compute prices for European options in milliseconds, improving by a factor 30 the performance, as shown in Section 5 .

\subsection{Conic pricing via quantization}

Bid and ask prices using Conic distortion functions has been studied in many works, as for example Leippold and Scharer (2017), Corcuera et al. (2012), Cherny and Madan (2009), Madan and Cherny (2010) and Madan and Schoutens (2017). In our work we take inspiration from the approach of Madan and Schoutens (2016, Chapter 5), where the authors use a Monte Carlo simulation technique in order to price the bid-ask spread. Instead of simulating the stock price paths, we use the quantization approach, as it gives better convergence results.

Let us consider the pricing of a call option with strike $K$, maturity $T$, with risk free interest rate $r$. The price can be approximated as

$$
\operatorname{call}_{K, T}=e^{-r T} \mathbb{E}\left[\left(e^{\log \left(S_{T}\right)}-K\right)^{+}\right] \approx \frac{1}{N} e^{-r T} \sum_{j=1}^{N}\left(e^{y_{j}^{*}}-K\right)^{+} .
$$

Let us recall that, as showed in Madan and Schoutens (2016, Chapter 5), when calculating the bid price of a Call option, it is important to make sure that higher weights of the Conic distortion function are linked to lower payoffs values, and lower weights are linked to higher payoff values.

Let us define payoff ${ }_{j}=\left(e^{y_{j}^{*}}-e^{k}\right)^{+}$, then payoff $_{1} \leq \cdots \leq$ payoff $_{N}$. Let us also underline that, since the distribution function $F$ is by definition non-decreasing, and since $u_{1} \leq u_{2} \leq \cdots \leq u_{N}$, then also the inverse points $y_{1}^{*}=F^{-1}\left(u_{1}\right) \leq \cdots \leq y_{N}^{*}=F^{-1}\left(u_{N}\right)$.

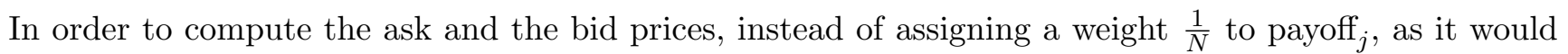
be in the risk neutral case, we choose a different approach. As studied in the Conic pricing literature, a 
distorted weight vector is assigned to the payoff vector. Let us call $\Psi$ a generic Conic distortion function. Then, according to Conic pricing theory, the element payoff ${ }_{j}$ is associated with the weight

$$
p_{j}^{*}=\Psi\left(\frac{j}{N}\right)-\Psi\left(\frac{j-1}{N}\right), \quad j=1 \ldots, N,
$$

for the bid price and

$$
\tilde{p}_{j}=\Psi\left(\frac{N-j+1}{N}\right)-\Psi\left(\frac{N-j}{N}\right), \quad j=1 \ldots, N,
$$

for the ask price. The bid-ask pricing of a call option in the uniform Fourier quantization approach is then

$$
\operatorname{call}_{K, T}^{\mathrm{bid}} \approx e^{-r T} \sum_{j=1}^{N}\left(e^{y_{j}^{*}}-K\right)^{+} p_{j}^{*}, \quad \operatorname{callask}_{K, T}^{\mathrm{ask}} \approx e^{-r T} \sum_{j=1}^{N}\left(e^{y_{j}^{*}}-K\right)^{+} \tilde{p}_{j} .
$$

\subsection{Error estimation}

In this subsection we present an a priori estimation of the error, when computing the price of a European Vanilla option. We assume that we are computing the risk neutral price of a Put option, the estimation of the error for a Call option is direct consequence of the put call parity.

Let us define the Put payoff $h(x)=\left(K-e^{x}\right)^{+}$, for a given strike $K$. The scope of this subsection is to give an estimation of

$$
\left|\mathbb{E}\left[h\left(F^{-1}(U)\right)\right]-\mathbb{E}\left[h\left(F^{-1}\left(U^{\Gamma_{N}^{*}}\right)\right)\right]\right|,
$$

where $U \sim U([0,1])$ and $U^{\Gamma_{N}^{*}}$ is its optimal N-quantization, as defined in Section 4.2 .

The first step involves showing that the function $u \rightarrow h\left(F^{-1}(u)\right)$ is Lipschitz. This is due to the fact that $F^{-1}$ is monotonically increasing, and that $h$ is monotonically decreasing and bounded. The function $u \rightarrow h\left(F^{-1}(u)\right)$ is then monotonically decreasing and bounded, and the derivative is also bounded, assuming that the density of the process has a smooth left tail. See Remark 4.2 for more details. This means that there exists a Lipschitz constant $[h ; F]_{\text {Lip }}$ such that

$$
\left|h\left(F^{-1}\left(u_{1}\right)\right)-h\left(F^{-1}\left(u_{2}\right)\right)\right| \leq[h ; F]_{\text {Lip }}\left|u_{1}-u_{2}\right|, \quad \forall u_{1}, u_{2} \in[0,1] .
$$

Let us underline the fact that the Lipschitz constant depends both on $h$ (therefore on the strike $K$ ) and on the distribution of the underlying. Using the results in Pagès (2015), the error in $(12)$ becomes

$$
\left|\mathbb{E}\left[h\left(F^{-1}(U)\right)\right]-\mathbb{E}\left[h\left(F^{-1}\left(U^{\Gamma_{N}^{*}}\right)\right)\right]\right| \leq[h ; F]_{\operatorname{Lip}}\left\|U-U^{\Gamma_{N}^{*}}\right\| .
$$

Using the results in Graf and Luschgy (2000, Example 4.17), we finally have that

$$
\left|\mathbb{E}\left[h\left(F^{-1}(U)\right)\right]-\mathbb{E}\left[h\left(F^{-1}\left(U^{\Gamma_{N}^{*}}\right)\right)\right]\right| \leq \frac{1}{4}[h ; F]_{\operatorname{Lip}} \frac{1}{N} .
$$

Remark 4.1. The result in (13) gives a quick understanding of why quantization can be used as an alternative to Monte Carlo methods in the computation of derivative prices. It is worth mentioning that this result is not an asymptotic estimation, as for example provided in Callegaro et al. (2018), where the authors study in detail the impact of the characteristic function on the convergence of the quantization error.

Remark 4.2. The derivative of $h\left(F^{-1}(u)\right)$, with $u \in[0,1]$ is

$$
\begin{cases}-\frac{\exp \left(F^{-1}(u)\right)}{f\left(F^{-1}(u)\right)} & \text { for } 0 \leq u \leq F(\ln (K)), \\ 0 & \text { for } F(\ln (K))<u \leq 1,\end{cases}
$$


where $f$ is the first derivative of $F$, i.e. the probability density function. In $u=0$ there might be explosion of the derivative of $h\left(F^{-1}(u)\right)$. In particular

$$
\lim _{u \rightarrow 0}-\frac{\exp \left(F^{-1}(u)\right)}{f\left(F^{-1}(u)\right)}=\frac{0}{0}
$$

Using L'Hopital rule, the limit is equal to

$$
\lim _{u \rightarrow 0}-\frac{\exp \left(F^{-1}(u)\right)}{f\left(F^{-1}(u)\right)}=\lim _{u \rightarrow 0}-\frac{\exp \left(F^{-1}(u)\right)}{f^{(n)}\left(F^{-1}(u)\right)},
$$

for every $n>1$, where $(n)$ indicates the $n$-th derivative. If the probability density function $f$ is regular enough to have one of its derivatives that is dominated by the exponential function at $-\infty$, then the first derivative of $h\left(F^{-1}(u)\right)$ is bounded in the interval $[0,1]$, and therefore $h\left(F^{-1}(u)\right)$ is Lipschitz.

\section{$5 \quad$ Numerical examples}

When considering the mid price of a derivative in presence of the characteristic function in closed form, a Fourier method as in Carr and Madan (1999) is the most used technique in both the academic and the practitioners world. The quantization method proposed in Section 4 can be seen as an alternative to Carr and Madan (1999) for the computation of mid prices, and a solution to the problem of pricing bid and ask prices, when the characteristic function of the underlying is known or easily computable. The quantization method previously developed is very general, in the sense that the pricing algorithm is not tailor-made for a particular model. Instead, as long as the characteristic function is known, then it is possible to price European options on every underlying. This allows us to price a huge set of models 1 .

\subsection{Pricing exercise}

We present here a numerical example for the pricing of a European option. We recall that the pricing of a call option in a quantization framework boils down to the dot product of the weights vector and of the payoff vector. In the case studied in Section 4 of Uniform Quantization, it boils down to computing the average of the payoff vector:

$$
\operatorname{call}_{K, T} \approx \frac{1}{N} e^{-r T} \sum_{j=1}^{N}\left(e^{y_{j}^{*}}-K\right)^{+},
$$

where $y_{j}^{*}$ are the solution of the system (8). We present here two examples for the Heston model, which is the most famous and most used stochastic volatility model. The application of the methodology to other models is straightforward.

\subsubsection{The Heston model}

The Heston model considers the following dynamics for the pair of price and volatility $(S, V)$ :

$$
\begin{aligned}
\frac{d S_{t}}{S_{t}} & =r d t+\sqrt{V_{t}}\left(\rho d W_{t}^{1}+\sqrt{1-\rho^{2}} d W_{t}^{2}\right), \\
d V_{t} & =\kappa\left(\theta-V_{t}\right)+\xi \sqrt{V_{t}} d W_{t}^{1},
\end{aligned}
$$

\footnotetext{
${ }^{1}$ A (non exhaustive) collection can be summarized as follow. Stochastic volatility: Heston model of Heston (1993), Bates model of Bates (1996), Bi Heston model of Christoffersen et al. (2009), Hull - White model of Hull and White (1987), Stein - Stein model of Stein and Stein (1991), Wishart model of Da Fonseca et al. (2008). Exponential Levy: Variance Gamma model of Madan et al. (1998), Normal Inverse Gaussian model of Barndorff-Nielsen (1997), CGMY model of Carr et al. (2002), Tempered Stable model of Kuchler and Tappe (2013). Non affine models: $\frac{3}{2}$ model of Platen (1997), $\frac{4}{2}$ model of Grasselli (2016).
} 
where $V$ denotes the instantaneous variance of the price process and it is modeled as a CIR process. Here $W^{1}$ and $W^{2}$ are two independent standard Brownian motions and $r$ is the interest rate. The parameters of the models are $\theta$ (the long-run average variance), $\kappa$ (the speed of the mean reversion of the variance), $\xi$ (the vol of vol parameter) and the correlation $\rho$.

We present here a numerical exercise, where we compare the price of call options with different strikes. The benchmark price is given by the algorithm developed in Carr and Madan (1999). The parameters of the Heston model are obtained via a market calibration on the DAX index as of date August, 28th 2008. The size of the quantization grid (1000 points) is chosen to have an average relative error under 10 basis points $(1 \mathrm{bp}=0.01 \%)$. The results are presented in Table 1, and show that the quantization algorithm is precise when pricing European options. It is also worth noting that the computational time makes the pricing algorithm almost instantaneous. By pricing algorithm here we consider the computation of the optimal uniform grid and the pricing of the option.

Figure 1 shows the convergence of the quantization pricing algorithm for an At The Money Call Option. It is worth noting that the quantization price is always smaller than the benchmark price. This has been noted and studied in Pagès (2015), and is motivated by the fact that the density of the original (benchmark) process dominates in in a convex way the density of the quantized process.

Figure 2 shows the computational cost for the computation of the quantization grids. As expected, apart from some numerical noise, the cost has a linear dependance with respect to the grid size. This is due to the fact that the system for the computation of the grids is solved via interpolation.

\begin{tabular}{|l|c|c|c|c|}
\hline Strike & Benchmark price & Quantization price & Relative error (bp) & ImpVol sqdiff $\left(10^{-8}\right)$ \\
\hline$K=80$ & 25.9677 & 25.9587 & 3.4800 & 14.4584 \\
$K=85$ & 22.2394 & 22.2316 & 3.4921 & 7.5735 \\
$K=90$ & 18.7952 & 18.7877 & 4.0037 & 5.4109 \\
$K=95$ & 15.6696 & 15.6632 & 4.0685 & 3.1682 \\
$K=100$ & 12.8884 & 12.8821 & 4.8749 & 2.6873 \\
$K=105$ & 10.4650 & 10.4585 & 6.1758 & 2.6531 \\
$K=110$ & 8.3985 & 8.3910 & 8.8745 & 3.5080 \\
$K=115$ & 6.6730 & 6.6667 & 9.4736 & 2.6618 \\
$K=120$ & 5.2601 & 5.2517 & 15.9185 & 5.1973 \\
\hline
\end{tabular}

Table 1: Pricing comparison between the benchmark price and the price obtained via quantization of a Call option for the Heston model with parameters $\kappa=2.3924, \xi=0.6903, V_{0}=0.0719, \theta=0.0929$, $\rho=-0.3210, r=0.04, T=1$. The quantization grids have size $\mathbf{N}=\mathbf{1 0 0 0}$, and the computational time is around 10 milliseconds. The benchmark price is computed using an FFT pricer.

\subsubsection{The Tempered Stable model}

The Tempered Stable model has been introduced in Cont et al. (1997), and assumes that the stock has the dynamics of an exponential Lévy process:

$$
S_{t}=S_{0} \exp \left[\left(r+\Gamma_{c}\right) t+X_{t}\right], \quad t \in[0, T],
$$

where $\Gamma_{c}$ is needed to ensure under the risk neutral measure and $X$ denotes a Lévy process associated with a tempered stable distribution. Tempered stable distributions form a six parameter family of infinitely divisible distributions. They include the Variance Gamma distributions of Madan et al. (1998), the bilateral Gamma distributions of Kuchler and Tappe (2008) and the CGMY distributions of Carr et al. (2002). Here we assume that $X \sim T S\left(c^{+}, \alpha^{+}, \lambda^{+}, c^{-}, \alpha^{-}, \lambda^{-}\right)$. The parameters $\lambda^{+}$and $\lambda^{-}$describe the rates of the tail decay, $\alpha^{+}$and $\alpha^{-}$involve positive and negative jumps, and $c^{+}$and $c^{-}$are the arrival rate of jumps of given size. 


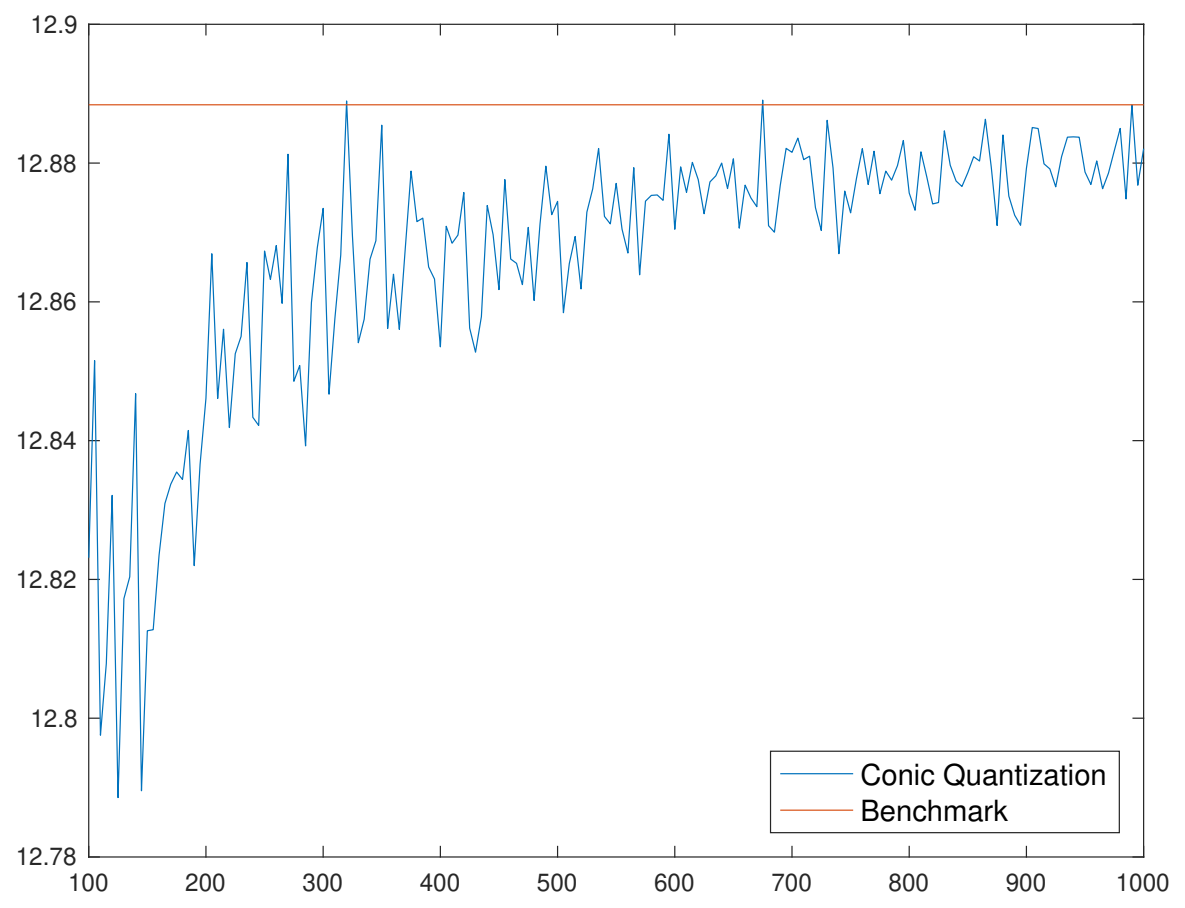

Figure 1: Price of an At The Money Call Option under the Heston model using the parameters of Table 1 for different choices of the quantization grid size. The benchmark price is given by the Carr Madan formula. The x-axis corresponds to the size of the quantization grid.

The Variance Gamma model, introduced by Madan et al. (1998), is an important sub case of the tempered stable family. In this framework, the Conic quantization approach is a generalization of the example that has been studied in Madan and Schoutens (2016).

We present here a case of Lévy processes in order to show the flexibility of the uniform quantization technique also when not considering processes where the volatility is stochastic and has an explicit SDE. The results presented in Table 2 are similar to the ones of Table 1, in terms of relative error and computational time. The parameters are taken from Cont et al. (1997). The benchmark is again given by the algorithm in Carr and Madan (1999), as the characteristic function is known in closed form.

\begin{tabular}{|l|c|c|c|c|}
\hline Strike & Benchmark price & Quantization price & Relative error $(\mathrm{bp})$ & ImpVol sqdiff $\left(10^{-7}\right)$ \\
\hline$K=80$ & 28.5214 & 28.4966 & 8.6903 & 8.0272 \\
$K=85$ & 25.2837 & 25.2592 & 9.7120 & 6.2545 \\
$K=90$ & 22.3119 & 22.2878 & 10.7988 & 5.0239 \\
$K=95$ & 19.6104 & 19.5870 & 11.9197 & 4.1266 \\
$K=100$ & 17.1775 & 17.1541 & 13.6605 & 3.7794 \\
$K=105$ & 15.0054 & 14.9824 & 15.3371 & 3.4267 \\
$K=110$ & 13.0812 & 13.0569 & 18.6174 & 3.7360 \\
$K=115$ & 11.3883 & 11.3643 & 21.0614 & 3.6304 \\
$K=120$ & 9.9072 & 9.8813 & 26.1757 & 4.3594 \\
\hline
\end{tabular}

Table 2: Pricing comparison between the benchmark price and the price obtained via quantization of a Call option for the Tempered Stable model with parameters $\alpha^{+}=1, \lambda^{+}=0.5, c^{+}=6, \alpha^{-}=1, \lambda^{-}=0.5$, $c^{-}=4, r=0.04, T=1$. The quantization grids have size $\mathbf{N}=\mathbf{1 0 0 0}$, and the computational time is around 35 milliseconds. The benchmark price is computed using a FFT pricer. 


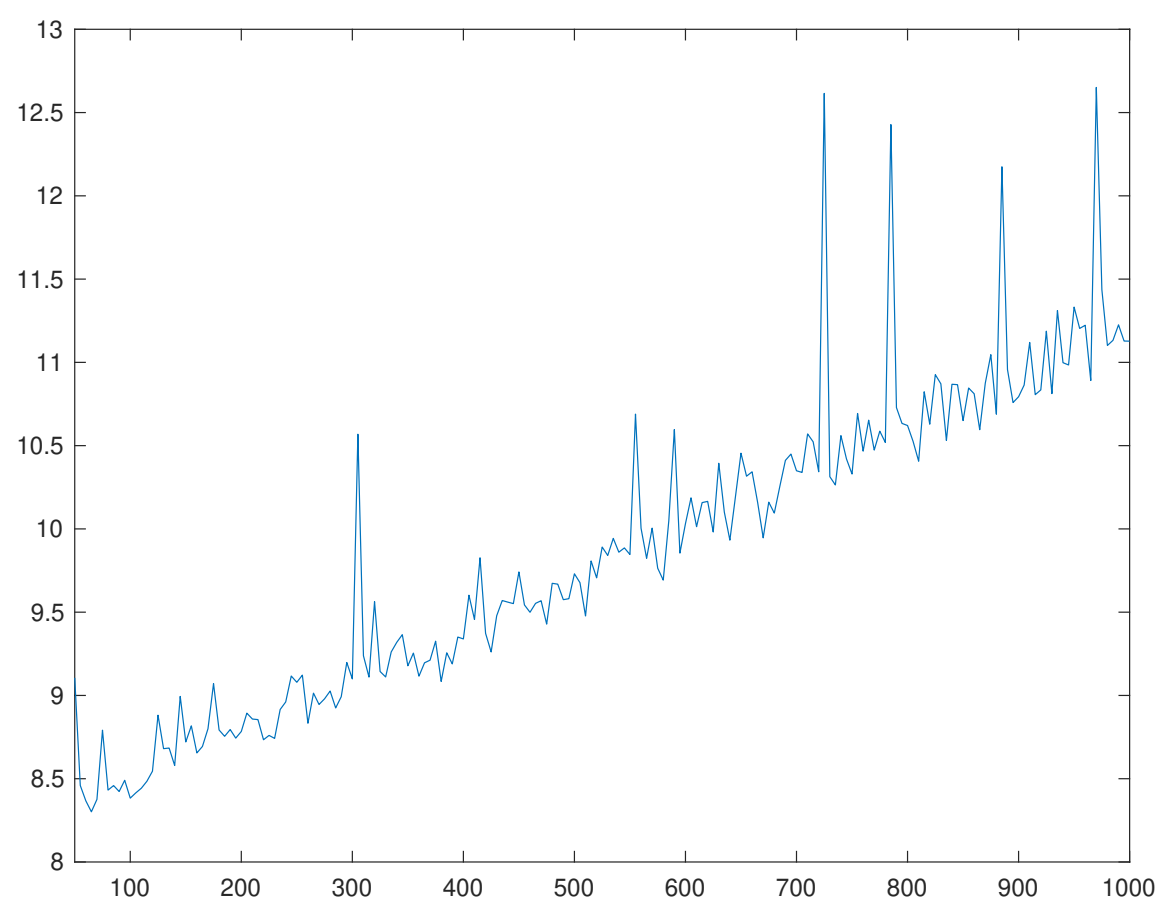

Figure 2: Computational time in milliseconds for the pricing of the quantization grids for the Heston model using the parameters of Table 1 . The x-axis corresponds to the size of the quantization grid.

The computational time in our examples prove that this method is precise and computationally comparable to a FFT approach, that we used as benchmark. We used two measures for the error. The

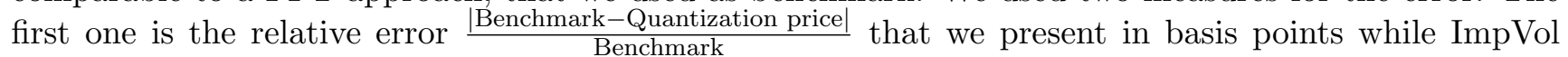
sqdiff is a measure that involves the implied volatility of the prices, i.e. $\left(\mathrm{impvol}_{\text {Benchmark }}-\mathrm{impvol}_{\text {Quantization }}\right)^{2}$, and is mostly used in the industry practice.

The two examples here study the behavior of the pricing of European call options for a given choice of the size $N$ of the quantization grids. Note that the computational time is composed by:

1. The solution of (8), which is the most computationally intense.

2. The finite sum in (9), which is just a finite sum and is immediate to compute.

The benchmark price is computed using a Fourier approach as in Carr and Madan (1999). This pricing exercise shows that the uniform Fourier quantization technique can price stochastic volatility models in a fast and precise way. The interesting part of this technique is that it can be easily implemented in a Conic pricing framework, as we will see later. Up to our knowledge, it is not possible to do the same using the technology of Carr and Madan (1999) for a generic model with a characteristic function in closed form.

\subsection{Conic quantization pricing}

Given the pricing algorithm developed in the previous sections, it is possible to recreate an implied volatility bid-ask smile, using the features of conic pricing theory. We choose the same model as one of the pricing exercises, the Heston model, and we use the generalized Conic distortion function MINMAXVAR (see Madan and Schoutens (2016) ) defined as

$$
\Psi_{\lambda, \gamma}(u)=1-\left(1-u^{\frac{1}{\lambda+1}}\right)^{1+\gamma}
$$


where both $\lambda$ and $\gamma$ are two real positive numbers. We present here a plot of the implied volatilities presenting the mid, the ask and the bid price. The implied volatilities of call options are on the left of the smile, while put options are on the right. The parameters $\lambda$ and $\gamma$ are chosen for this numerical example to be $\gamma=\lambda=0.01$. Figure 3 shows the behaviour of a representative bid-ask smile when the underlying model is given by the Heston model.

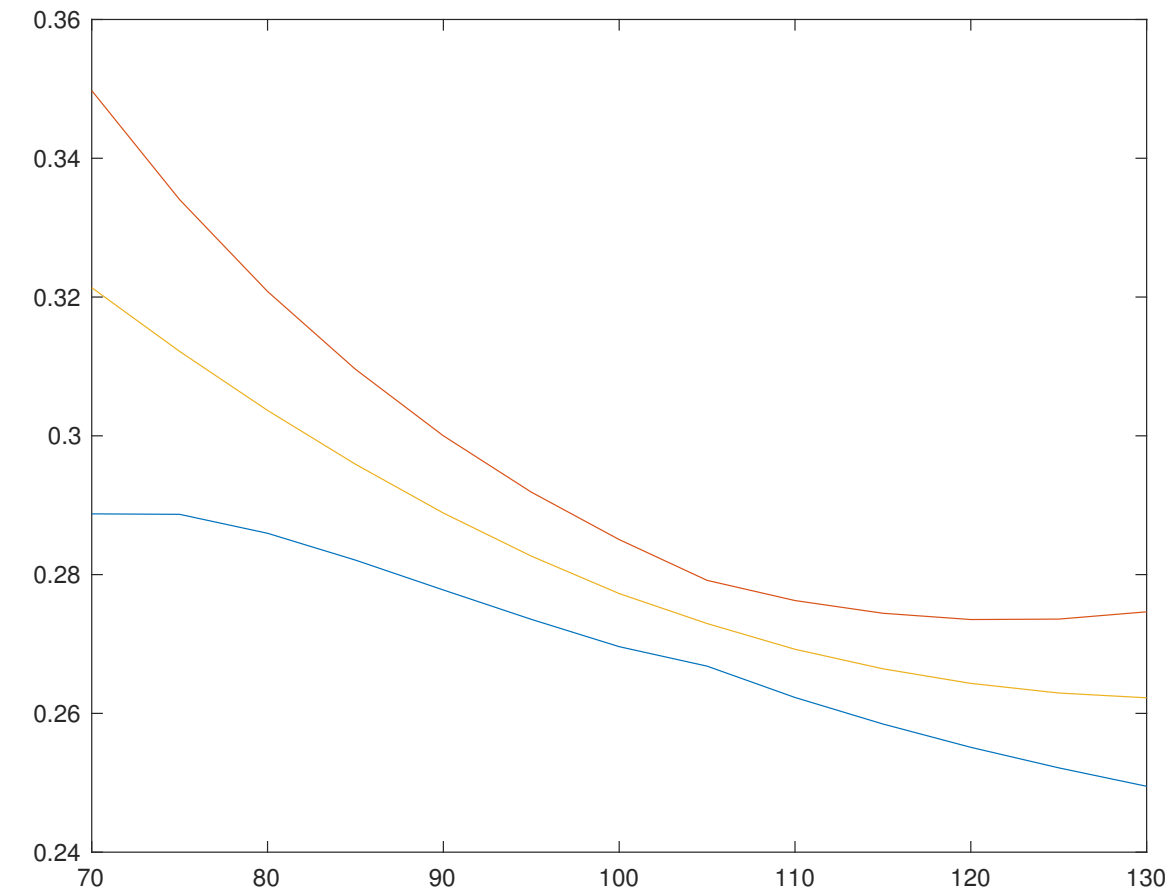

Figure 3: Bid - Ask smile using the MINMAXVAR Conic distortion function with parameter $\lambda=0.01$ in the Heston model as in Table 1 and $N=1000$ points for the quantization grid. The x-axis corresponds to the strike of the Vanilla option, the y-axis is the corresponding implied volatility.

\begin{tabular}{|c|c|c|c|}
\hline Strike & Bid price & Mid price & Ask price \\
\hline$K=70$ & 33.6578 & 34.1099 & 34.5652 \\
$K=75$ & 29.4982 & 29.9317 & 30.3687 \\
$K=80$ & 25.5474 & 25.9587 & 26.3739 \\
$K=85$ & 21.8460 & 22.2316 & 22.6215 \\
$K=90$ & 18.4307 & 18.7877 & 19.1492 \\
$K=95$ & 15.3372 & 15.6632 & 15.9939 \\
$K=100$ & 12.5886 & 12.8821 & 13.1803 \\
$K=105$ & 10.1982 & 10.4585 & 10.7236 \\
$K=110$ & 8.1634 & 8.3910 & 8.6234 \\
$K=115$ & 6.4701 & 6.6667 & 6.8677 \\
$K=120$ & 5.0840 & 5.2517 & 5.4237 \\
$K=125$ & 3.9706 & 4.1123 & 4.2579 \\
$K=130$ & 3.0908 & 3.2096 & 3.3319 \\
\hline
\end{tabular}

Table 3: Bid, Mid and Ask prices using the MINMAXVAR Conic distortion function with parameter $\lambda=0.01$ in the Heston model as in Table 1 and $N=1000$ points for the quantization grid. 


\begin{tabular}{|c|c|}
\hline Parameters & Conic Quantization \\
\hline$\kappa$ & 2.2299 \\
$\xi$ & 0.4574 \\
$\rho$ & -0.6452 \\
$\theta$ & 0.0308 \\
$V_{0}$ & 0.0163 \\
$\lambda$ & 0.0001 \\
$\gamma$ & 0.0108 \\
\hline Res Norm & $1.8460 \times 10^{-4}$ \\
\hline
\end{tabular}

Table 4: Heston model calibrated on a book of bid and ask vanillas on the SP500 index as of date July, $11^{\text {th }} 2017$. The book includes 8 maturities (from 6 weeks to 3 years) with 18 strikes, for a total of 288 options. Res Norm indicates the average square error on implied volatilities.

\section{Calibration on market data}

The possibility of computing the bid-ask implied volatility smile opens the doors to a more general approach towards the calibration of market data. For this calibration study we stick to the Heston model as for the underlying model. The choice of the model is given by the fact that it is quite parsimonious, as it consists of only 5 parameters. It is possible to use other models, as the uniform quantization technique is flexible enough to consider mode involved models.

The market data that we consider in this Section are taken from the SP500 index as of date July, $11^{\text {th }}$ 2017, and consist of Put and Call bid-ask prices for different strikes and maturities. We will consider two approaches when calibrating using the uniform quantization technique and the conic framework,.

\subsection{Global fit}

A first approach to the calibration of the bid-ask smile is considering a generalized MINMAXVAR Conic distortion function where the parameters $\lambda$ and $\gamma$ are fixed for every strike and maturity. This approach gives a global value of this liquidity parameters in the market.

The book of options considered includes 8 maturities with 18 different strikes, for a total of 288 options, since we are considering both ask and bid prices in the calibration routine. The calibration algorithm that we implemented consists in finding the minimum of the average square implied volatility error, i.e. we minimize

$\frac{1}{\#_{\text {strikes }} \times \#_{\text {maturities }}} \sum_{i=1}^{\#_{\text {strikes }}} \sum_{j=1}^{\#_{\text {maturities }}}\left[\left(I V_{i, j}^{\mathrm{bid}, \mathrm{mkt}}-I V_{i, j}^{\mathrm{bid}, \mathrm{mod}}(\text { param })\right)^{2}+\left(I V_{i, j}^{\text {ask,mkt }}-I V_{i, j}^{\text {ask,mod }}(\text { param })\right)^{2}\right]$,

where $I V_{i, j}^{\text {bid,mkt }}$ and $I V_{i, j}^{\text {ask,mkt }}$ are the bid and ask implied volatility taken from market data, while $I V_{i, j}^{\text {bid,mod }}$ (param) and $I V_{i, j}^{\text {ask,mod }}$ (param) are the bid and ask implied volatility computed using the conic quantization technique, where param contains both the model parameters and the Conic distortion function parameters. In Table 4 we present the results of the calibration exercise, while Figure 4 and 5 provide the squared errors for both bid and ask implied volatilities. The results are interesting in the sense that the fit using this technique is very good, in particular close to at-the-money options. We could have considered a term structure for the parameters of the Heston model, in order to guarantee a better fit to market data, but the choice of constant parameters gives a much quicker and more straightforward analysis of the calibration results.

\subsection{Implied liquidity}

The choice of having constant parameters for the distortion function can be relaxed in order to have a more flexible fit to market data. It is then possible to consider the calibration of the bid-ask spread 


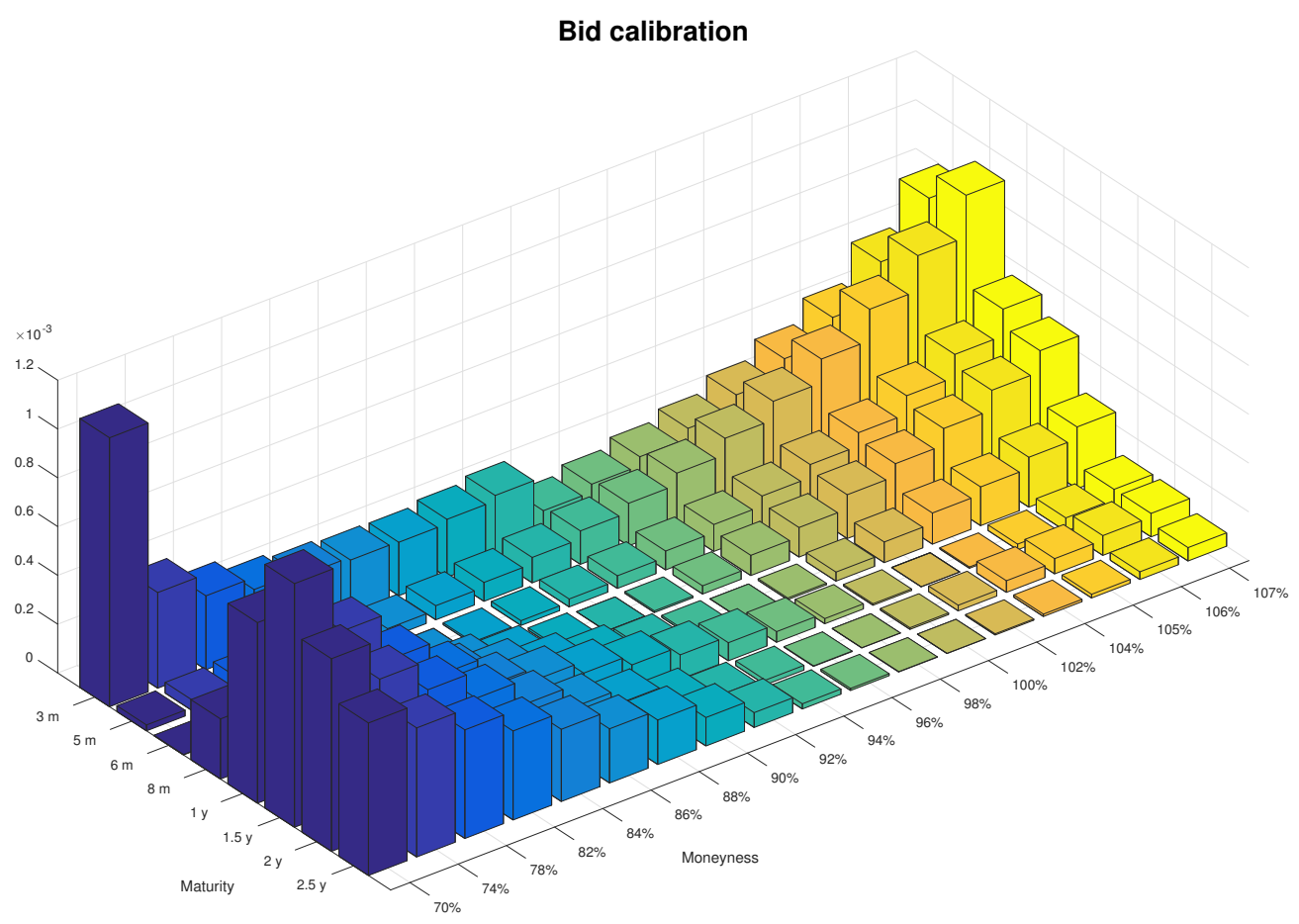

Figure 4: Plot of the square of the difference between the calibrated model implied volatilities and the market implied volatilities on bid prices, for all the 18 strikes and the 8 maturities of the book of vanillas on the SP500 index. 


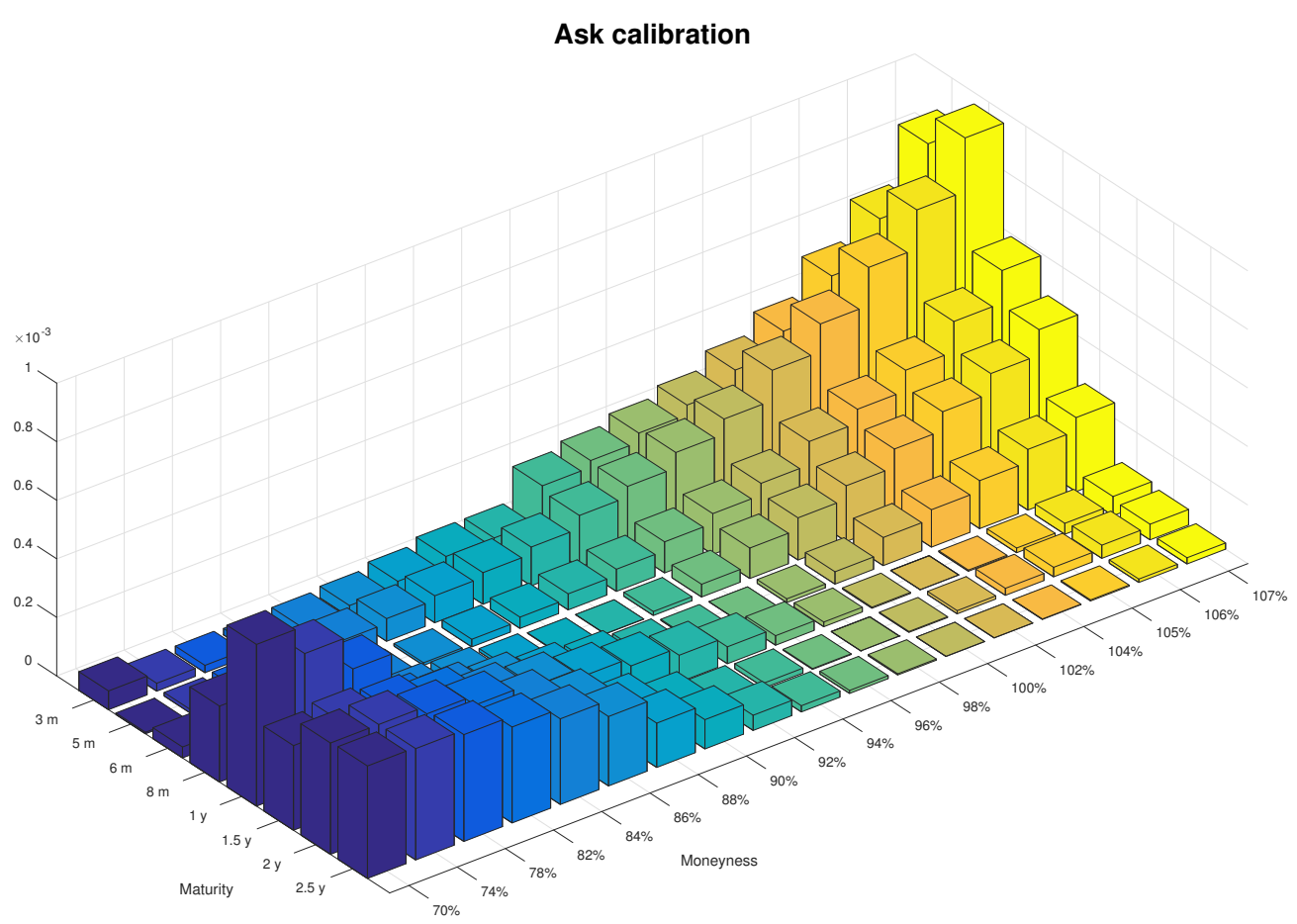

Figure 5: Plot of the square of the difference between the calibrated model implied volatilities and the market implied volatilities on ask prices, for all the 18 strikes and the 8 maturities of the book of vanillas on the SP500 index. 
under a different perspective. In this calibration exercise we use the usual MINMAXVAR Conic distortion function (i.e. the generalized function with $\gamma=\lambda$ ), and we assume that it is possible to calibrate a different $\lambda$ for every strike and every maturity in the book. We define the market implied liquidity as the parameter $\lambda$ which, for every strike and every maturity, minimizes the difference between the model ask/bid prices and the market prices.

In this calibration study, the calibration routine is done on mid, bid and ask prices, and in particular the distortion parameters $\lambda_{K, T}$ are obtained minimizing the distance between the model bid ask prices and the market bid ask prices.

This calibration approach is more flexible, and permits to have a better fit of the volatility smiles. In analogy with the implied volatility surface, we call the surface composed by $\lambda_{K, T}$ the implied liquidity surface of the market. Pay attention that this surface depends on the choice of the model, in this case the Heston model, and on the choice of the Conic distortion function, in this case the MINMAXVAR function. With different models and Conic distortion function we could expect a different shape of different levels of implied liquidity. Figure 6 shows the implied volatility surface of the market. It shows that the implied liquidity is decreasing for increasing values of the maturity and of the moneyness.

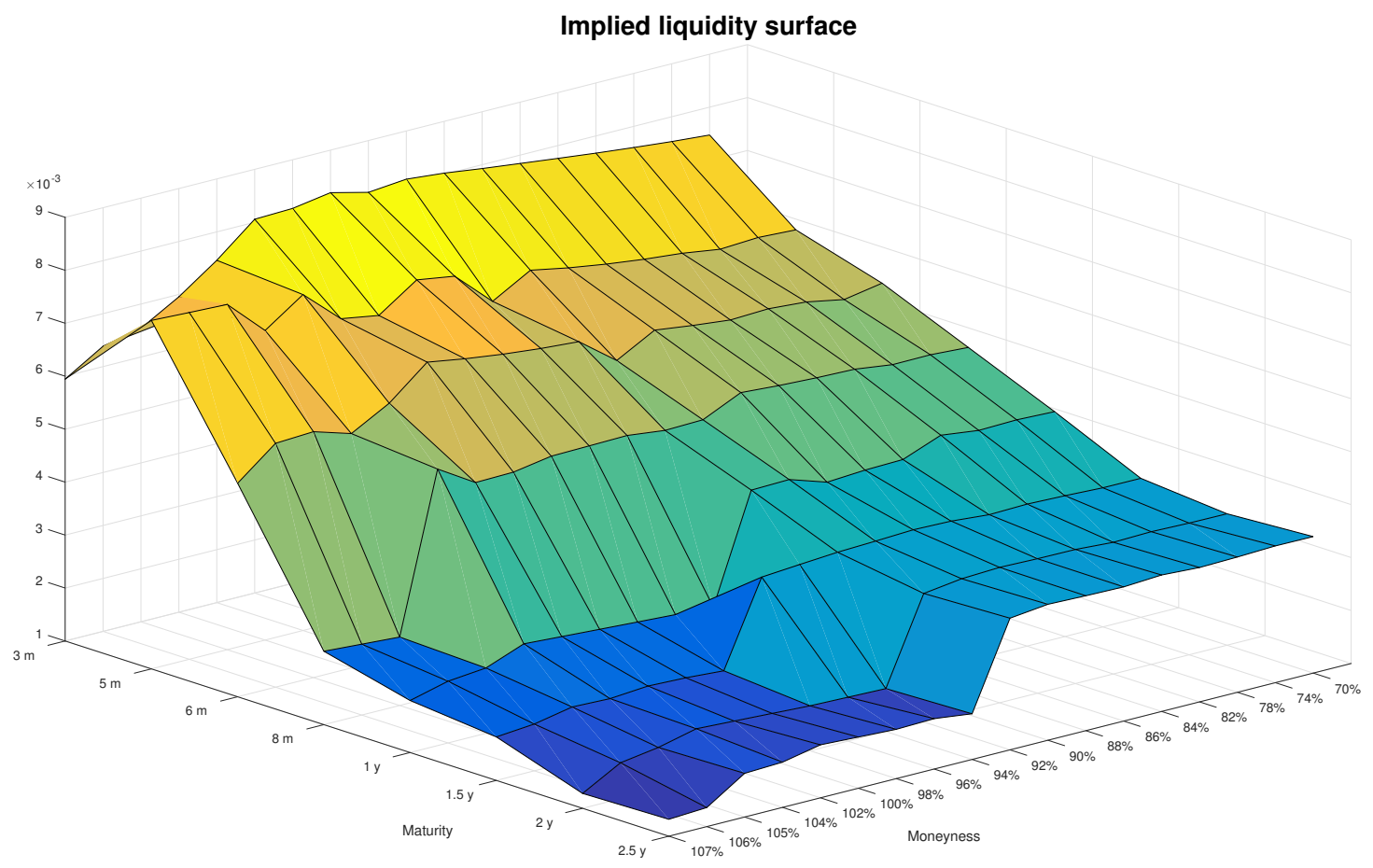

Figure 6: Plot of the implied liquidity surface for the SP500 index as of date July, $11^{\text {th }} 2017$ under the Heston model and the MINMAXVAR Conic distortion function.

\section{Conclusion}

The presence of liquidity in the market of derivatives is an important feature that has to be taken into account when calibrating a model to financial data. Indeed, the mid price is a price that does not exist in reality, so calibration exercises can be biased. The theory of two prices and the conic finance theory tackle this issues and gives a computational framework for the pricing of the bid and ask spread for a European derivative. The uniform quantization algorithm developed in this paper allows us to consider 
the liquidity of the market and the presence of stochastic volatility in the same pricing mechanism, and allows to calibrate in an efficient and flexible way the implied liquidity surface given by the market.

\section{References}

Albrecher, H., Guillaume, F., and Schoutens, W. (2013). Implied liquidity: Model sensitivity. Journal of Empirical Finance, 23:48 - 67.

Artzner, P., Delbaen, F., Eber, J.-M., and Heath, D. (1999). Coherent measures of risk. Mathematical Finance, 9(3):203-228.

Barles, G. and Soner, H. M. (1998). Option pricing with transaction costs and a nonlinear black-scholes equation. Finance and Stochastics, 2:369-397.

Barndorff-Nielsen, O. E. (1997). Normal Inverse Gaussian Distributions and Stochastic Volatility Modelling. Scand. J. Statist., 24:1-13.

Bates, D. S. (1996). Jumps and Stochastic Volatility: Exchange Rate Processes Implicit in Deutsche Mark Options. The Review of Financial Studies, 9:69-107.

Callegaro, G., Fiorin, L., and Grasselli, M. (2015). Quantized calibration in local volatility. Risk Magazine, 28(4):62 - 67 .

Callegaro, G., Fiorin, L., and Grasselli, M. (2016). Pricing via recursive quantization in stochastic volatility models. Quantitative Finance, Issue 6:855-872.

Callegaro, G., Fiorin, L., and Grasselli, M. (2018). Quantization meets fourier: a new technology for pricing options. Annals of Operations Research.

Carr, P., Geman, H., Madan, D., and Yor, M. (2002). The fine structure of asset returns: An empirical investigation. The Journal of Business, 75(2):305-332.

Carr, P. and Madan, D. B. (1999). Option valuation using the fast fourier transform. Journal of Computational Finance, 2:61-73.

Cherny, A. and Madan, D. (2009). New measures for performance evaluation. The Review of Financial Studies, 22(7):2571-2606.

Christoffersen, P., Heston, S., and Jacobs, K. (2009). The Shape and Term Structure of the Index Option Smirk: Why Multifactor Stochastic Volatility Models Work So Well. Management Science, 55(12):1914-1932.

Cont, R., Bouchaud, J.-P., and Potters, M. (1997). Scaling in financial data: Stable laws and beyond. Scale Invariance and Beyond, Dubrulle, B., Graner, F., and Sornette, D., eds., Springer. Berlin, 1997.

Corcuera, J. M., Guillaume, F., Madan, D. B., and Schoutens, W. (2012). Implied liquidity: towards stochastic liquidity modelling and liquidity trading. Int. J. Portfolio Analysis and Management, 1:80 $-91$.

Cvitanic, J. and Karatzas, I. (1996). Hedging and portfolio optimization under transaction costs: a martingale approach. Mathematical Finance, 6(2):133-165.

Da Fonseca, J., Grasselli, M., and Tebaldi, C. (2008). A multifactor volatility heston model. Quantitative Finance, 8(6):591-604.

Davis, M., Panas, V., and Zariphopoulou, T. (1993). European option pricing with transaction costs. SIAM Journal on Control and Optimization, 31(2):470-493. 
Fiorin, L., Pagès, G., and Sagna, A. (2018). Product markovian quantization of a diffusion process with applications to finance. Methodology and Computing in Applied Probability.

Graf, S. and Luschgy, H. (2000). Foundations of quantization for probability distributions. Springer, New York.

Grasselli, M. (2016). The 4/2 Stochastic Volatility Model. Mathematical Finance, forthcoming.

Heston, S. L. (1993). A closed-form solution for options with stochastic volatility with applications to bond and currency options. Review of Financial Studies, 6:327-343.

Hull, J. and White, A. (1987). The Pricing of Options on Assets with Stochastic Volatilities. The Journal of Finance, 42:281-300.

Kuchler, U. and Tappe, S. (2008). Bilateral gamma distributions and processes in financial mathematics. Stochastic Processes and their Applications, 118(2):261 - 283.

Kuchler, U. and Tappe, S. (2013). Tempered stable distributions and processes. Stochastic Processes and their Applications, 123(12):4256 - 4293.

Leippold, M. and Scharer, S. (2017). Discrete-time option pricing with stochastic liquidity. Journal of Banking and Finance, 75:1 - 16.

Madan, D. and Schoutens, W. (2016). Applied Conic Finance. Cambridge.

Madan, D. B., Carr, P. P., and Chang, E. C. (1998). The variance gamma process and option pricing. European finance review, 2(1):79-105.

Madan, D. B. and Cherny, A. (2010). Markets as counterparty: an introduction to conic finance. International Journal of Theoretical and Applied Finance, 13(08):1149-1177.

Madan, D. B. and Schoutens, W. (2017). Conic option pricing. The Journal of Derivatives, 25(1):10-36.

McWalter, T. A., Rudd, R., Kienitz, J., and Platen, E. (2018). Recursive marginal quantization of higher-order schemes. Quantitative Finance, 18(4):693-706.

Pagès, G. (2015). Introduction to vector quantization and its applications for numerics. ESAIM: proceedings and surveys, 48:29-79.

Pagès, G. and Sagna, A. (2015). Recursive marginal quantization of the Euler scheme of a diffusion process. Applied Mathematical Finance, 22(5):463-498.

Platen, E. (1997). A non-linear stochastic volatility model. Financial Mathematics Research Report No. FMRR 005-97, Center for Financial Mathematics, Australian National University, Canberra.

Shephard, N. G. (1991). From characteristic function to distribution function: A simple framework for the theory. Econometric Theory, 7(4):519-529.

Shreve, S. E. and Soner, H. M. (1994). Optimal investment and consumption with transaction costs. Ann. Appl. Probab., 4(3):609-692.

Stein, E. M. and Stein, J. C. (1991). Stock price distributions with stochastic volatility: an analytic approach. Review of Financial Studies, (4):727 - 752. 